\title{
An ergodic theorem for iterated maps
}

\author{
JOHN H ELTON
}

School of Mathematics, Georgia Institute of Technology, Atlanta, Georgia 30332, USA

(Recevved 10 Aprll 1986 and revised 11 August 1986)

Abstract Consider a Markov process on a locally compact metric space arising from iteratively applyıng maps chosen randomly from a finite set of Lipschitz maps which, on the average, contract between any two points (no map need be a global contraction) The distribution of the maps is allowed to depend on current position, with mild restrictions Such processes have unique stationary initial distribution [BE], [BDEG]

We show that, starting at any point, time averages along trajectories of the process converge almost surely to a constant independent of the starting point This has applications to computer graphics

\section{Introduction}

Let $(X, d)$ be a metric space in which sets of finte diameter are relatively compact. Let $w_{1} X \rightarrow X$ be Lipschitz maps, with $d\left(w_{1} x, w_{2} y\right) \leq s_{1} d(x, y)$ for $x, y$ in $X, \imath=$ $1, \quad, N$ A good example is affine maps on $\mathbb{R}^{n}$ Let $p_{1} X \rightarrow[0,1]$ such that $p_{t}(x) \geq 0$ and $\sum_{t=1}^{N} p_{t}(x)=1$, and assume that the $p_{t}$ 's are continuous Define a Markov transition probability by

$$
p(x, B)=\sum_{i=1}^{N} p_{t}(x) 1_{B}\left(w_{t} x\right)
$$

This is the probability of transfer from $x \in X$ into the Borel set $B$ Intuitively, pick a number $\imath$ between 1 and $N$ according to the distribution $p_{t}(x)$ and go to $w_{r} x$

Such processes have been discussed in many places under the assumption that the maps are contractions and usually that the $p_{\imath}$ 's are constants [BD], [DF], [DS], $[\mathbf{H}],[\mathbf{K}]$ (Karlın [K] discussed variable $p_{\imath}$ 's ) It was shown recently [BE], [BDEG] that none of the $w_{t}$ 's need be contractions, but that if there is contraction 'on the average' between any two points, $1 \mathrm{e}$

$$
\prod_{i=1}^{N} d\left(w_{i} x, w_{i} y\right)^{p_{t}(x)} \leq r d(x, y) \quad \forall x, y, \text { where } r<1,
$$

and if the $p_{i}$ 's are bounded away from 0 and have moduli of continuity $\phi_{1}$ satisfying Dini's condition ( $1 \mathrm{e} \phi_{t}(t) / t$ is integrable over $(0, \alpha)$ for some $\left.\alpha>0\right)$, then there is a unique, attractive stationary initial probability distribution $\mu$ for the process This means

$$
V \mu(B)=\int p(x, B) d \mu(x)=\mu(B)
$$


for all Borel sets $B$ and, for all initial probability distributions $\nu, V^{n} \nu$ converges in distribution (that is, weakly) to $\mu, 1$ e $\int f d V^{n} \nu \rightarrow \int f d \mu$ for all bounded continuous functions $f$ on $X$

Our object is to show that starting at any $x \in X$, the trajectories (orbits) of the process converge in distribution to $\mu$ almost surely By this we mean that for almost all trajectories $x, x_{1}, x_{2}$, of the process starting at $x$, the time averages

$$
\frac{1}{n+1} \sum_{k=0}^{n} f\left(x_{k}\right)
$$

converge to $\int f d \mu$ for all bounded continuous $f$, or in yet other terms, the empincal distribution

$$
\nu_{n}=\frac{1}{n+1} \sum_{k=0}^{n} \delta_{x_{k}}
$$

of the first $n+1$ points along the trajectory converges weakly to $\mu$ as $n \rightarrow \infty$

Let us explain why this is important It follows (see Lemma 1) from the classical pointwise ergodic theorem that for $\mu$-almost all $x \in X$, almost all trajectories starting at $x$ converge in distribution to $\mu$ (in the sense just explained) But in applications to computer graphics, for example (see [BD]) we may have no way of choosing the starting $x$ according to the measure $\mu$, in fact, the idea is to start at some $x$ and let a computer-generated realization of the process 'draw a picture' of $\mu$

A special case of this result, when the maps $w_{\imath}$ are contractions with a special disjointness condition, and the $p_{i}$ 's are constants, was stated already by Diaconis and Shashahanı [DS] Most of the difficulty of our proof arises from having non-contractions and variable $p_{i}$ 's

First we prove a general lemma about Markov processes, and then we state and prove the main theorem, using a martıngale argument

\section{Markov processes with unique stationary distribution}

Let $(X, \mathscr{F})$ be an arbitrary measurable space, and let $p(), X \times \mathscr{F} \rightarrow[0,1]$ be a transition probability, 1 e $p(x$,$) is a probability measure for each x$, and $p(, A)$ is a measurable function for all $A \in \mathscr{F}$ A (discrete-tıme) stochastic process $\left\{Z_{n}, n=\right.$ $0,1, \quad\}$ with values in $X$ is called a Markov process with transition probability $p$ if

$$
P\left\{Z_{n+1} \in A \mid Z_{0}=z_{0}, \quad, Z_{n}=z_{n}\right\}=p\left(z_{n}, A\right) \text { a s }
$$

$X$ is called the state space Define the operator $V$ on finite measures by

$$
V \nu(A)=\int p(x, A) d \nu(x), \quad A \in \mathscr{F}
$$

A probability measure $\mu$ is called a stationary initıal distribution if $V \mu=\mu$ If $\mu$ is a stationary initial distribution and if $Z_{0}$ has distribution $\mu$, then $\left\{Z_{n}\right\}$ will be a stationary stochastic process Assume for the rest of this paragraph that $Z_{0}$ has a stationary initial distribution so that $\left\{Z_{n}\right\}$ is a stationary process $A$ is called an invariant event if there exists $C \in \mathscr{F}_{\infty}$ such that $A=\left\{\left(Z_{h}, Z_{k+1}, \quad\right) \in C\right\}$ for all $k \geq 0$, where $\mathscr{F}_{\infty}$ is the $\sigma$-algebra in $X^{\infty}$ generated by measurable cylinders $A$ is called almost invariant if there is an invariant event $B$ so that $P(A \triangle B)=0$ Let $\mathscr{I}$ denote 
the $\sigma$-algebra of almost invariant events The process $\left\{Z_{n}\right\}$ is called ergodic if for every $A \in \mathscr{I}, P(A)=0$ or 1 A reference for the above definitions is [D]

The next lemma is surely known, but we were unable to find a statement of it for general Markov processes We did find it stated in [FK] for a special case In any case, it follows very easily from well-known results

LEMMA 1 If $\mu$ is the unique stationary initial distribution (or just an extreme point of the set of stationary initial distributions), then the process $\left\{Z_{n}\right\}$ with $Z_{0}$ having distribution $\mu$ is ergodic

Proof If not, there is $A \in \mathscr{I}$ with $0<P(A)<1$ Then there exists $C \in \mathscr{F}$ such that $A=\left\{Z_{n} \in C\right\}$ a e for all $n \geq 0$, since $\left\{Z_{n}\right\}$ is a stationary Markov process [see S] Define

$$
\nu(B)=\mu(B \cap C) / \mu(C) \text { and } \lambda(B)=\mu(B \cap \sim C) / \mu(\sim C)
$$

(note that $\mu(C)=P(A)$ and $\mu(\sim C)=P(\sim A)$ ) Then $\mu=\mu(C) \nu+\mu(\sim C) \lambda$, so the proof will be completed by showing that $\nu$ (and hence $\lambda$ ) is a stationary initial distribution, since clearly $\nu \neq \lambda$

Now

$$
\begin{aligned}
\nu(B) & =P\left(Z_{1} \in B \cap C\right) / \mu(C) \\
& =P\left(\left(Z_{1} \in B\right) \cap\left(Z_{1} \in C\right)\right) / \mu(C) \\
& =P\left(\left(Z_{1} \in B\right) \cap\left(Z_{0} \in C\right)\right) / \mu(C) \\
& =\frac{1}{\mu(C)} \int_{C} P\left(Z_{1} \in B \mid Z_{0}=z\right) d \mu(z) \\
& =\frac{1}{\mu(C)} \int_{C} p(z, B) d \mu(z) \\
& =\int p(z, B) d \nu(z),
\end{aligned}
$$

since clearly $d \nu / d \mu=(1 / \mu(C)) 1_{C}$ But this says that $\nu$ is a stationary initial distribution

Remark The processes discussed in the introduction and the next section are not what is called indecomposable in [B] and Markov ergodic in [S], as the following simple example shows, so we could not quote the theorems in those references for our application

Example Let $X=[0,1], w_{1} x=\frac{1}{2} x, w_{2} x=\frac{1}{2}+\frac{1}{2} x, p_{t}=1 / 2, l=1,2$ Then all trajectories startıng at a rational number in $[0,1]$ stay in the rationals, and all trajectories starting at an irrational number in $[0,1]$ stay in the irrationals Thus the process is not indecomposable/Markov ergodic as defined in [B], [S] (some people use the word 'indecomposable' differently) However, the process $t s$ ergodic, since there is a unique stationary initial distribution

\section{Main results}

Let $\Omega=N^{\infty}=\left\{\left(t_{1}, l_{2}, \quad\right) 1 \leq l_{j} \leq N\right.$ and $l_{j}$ is an integer for each $\left.J\right\}$ Let $\mathscr{A}$ be the $\sigma$-algebra generated by the cylinders in $\Omega$ 
Return now to the setup of the introduction For each $x \in X$, let $P_{x}$ be the probability measure on $\mathscr{A}$ defined on cylinders by

$$
P_{x}\left(\left(l_{1}, t_{2}, \quad, l_{n}\right)\right)=p_{i_{1}}(x) p_{t_{2}}\left(w_{t_{1}} x\right) p_{t_{3}}\left(w_{t_{2}} w_{r_{1}} x\right) \quad p_{i_{n}}\left(w_{t_{n-1}} \quad w_{t_{1}} x\right)
$$

(we abuse notation by writing $P_{x}\left(\left(l_{1}, l_{2}, \quad, l_{n}\right)\right)$ when we mean $P_{x}\left(\left\{l_{1}, l_{2}, \quad, l_{n}\right)\right\} \times$ $N \times N \times N \times \quad$ )) It is clear this is precisely the probability measure for realizations of the Markov process starting at $x$ That is, if we consider a Markov process $\left\{Z_{n}, n=0,1, \quad\right\}$ with state space $X$ and transition probability $p$ as given in the introduction, then

$$
\begin{aligned}
& P\left(\left(Z_{0}, Z_{1}, \quad\right) \in B \mid Z_{0}=x\right) \\
& \quad=P_{x}\left\{\left(t_{1}, t_{2}, \quad\right)\left(x, w_{t_{1}} x, w_{t_{2}} w_{t_{1}} x, \quad\right) \in B\right\}
\end{aligned}
$$

for every $B \in \mathscr{F}_{\infty}$

THEOREM Suppose there exists $r<1$ such that

$$
\prod_{i=1}^{N} d\left(w_{t} x, w_{l} y\right)^{p_{l}(x)} \leq r d(x, y) \quad \forall x, y \text { in } X
$$

Assume there is $\delta>0$ such that $p_{i}(x) \geq \delta$ for all $x$ and 1 , and that the modult of continuty of the $p_{i}^{\prime}$ 's satisfy Dini's condition Let $\mu$ be the unique stationary inttal distribution for the Markov process described above (see [BDEG]) Then for every $x$ in $X$, there exists $G_{x} \subset \Omega$ such that $P_{x}\left(G_{x}\right)=1$ and for $\left(t_{1}, t_{2}, \quad\right) \in G_{x}$, we have

$$
\frac{1}{n+1} \sum_{k=0}^{n} f\left(w_{i_{k}} \quad w_{i_{1}} x\right) \rightarrow \int f d \mu
$$

for all $f \in C(X)$, that is, almost all trajectories $x, w_{t_{1}} x, w_{t_{2}} w_{1_{1}} x, \quad$ starting at $x$ converge in distribution to $\mu$ (in the sense explained in the introduction)

COROLlary 1 Let $\nu$ be any probabulity measure, and let $\left\{Z_{n}\right\}$ be the Markov process with initial distribution $\nu \overline{\text { and }}$ transition probability as above Assume the hypotheses of the Theorem Then for all $f \in C(X)$,

$$
\frac{1}{n+1} \sum_{k=0}^{n} f\left(Z_{k}\right) \rightarrow \int f d \mu \text { as }
$$

Remark It is shown in [FK] that Corollary 1 holds, in case $X$ is a compact metric space, for a general transition probability for which it is only required that $x \mapsto p(x$, is continuous with the measures being given the $w^{*}$-topology, and that there is a unique stationary initial distribution

Corollary 2 If $B \subset X$ is such that $\mu(\partial B)=0$, then for any $x \in X$, if $\left(l_{1}, l_{2}, \quad\right) \in G_{x}$, the average amount of time the trajectory spends in $B$ converges to $\mu(B)$, that is,

$$
\lim _{k \rightarrow \infty} \frac{\#\left\{j \quad 0 \leq J \leq k, w_{i_{j}} \quad w_{i_{1}} x \in B\right\}}{k+1}=\mu(B)
$$

Th1s follows from a well-known consequence of weak convergence, and generalizes a statement of [DS]

We prove two lemmas and then the Theorem and Corollary 1 The first lemma uses a martıngale argument 
Lemma 2 Let $x, y \in X, x \neq y$ Assume the hypotheses of the theorem Let $r<r_{1}<1$

(1) For all $\varepsilon>0$, there exist $n_{\varepsilon}$ and $S \subset \Omega$ with $P_{x}(S)<\varepsilon$ such that

except for $\left(t_{1}, t_{2}, \quad\right)$ in $S$,

$$
n \geq n_{\varepsilon} \Rightarrow d\left(w_{i_{n}} \quad w_{i_{1}} x, w_{i_{n}} \quad w_{i_{z}} y\right) \leq r_{1}^{n} d(x, y)
$$

(11) $\lim _{n \rightarrow \infty} d\left(w_{i_{n}} \quad w_{i_{1}} x, w_{i_{n}} \quad w_{i_{1}} y\right)=0$ as $-P_{x}$

Proof Let $s=\max \left\{s_{i}, l=1, \quad, N\right\} W$ log assume $s \geq 1$ Define random variables $X_{n}$ on $\Omega$ by

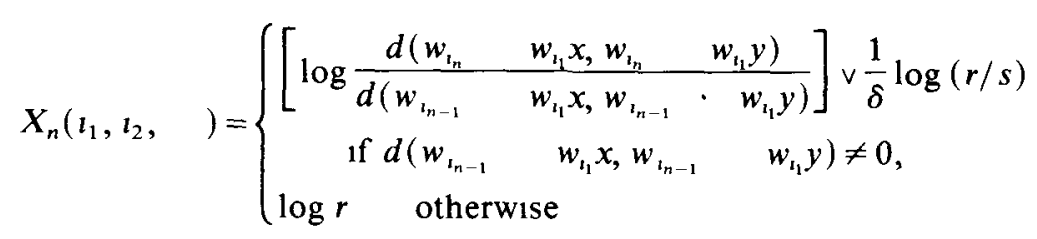

The purpose of the $(1 / \delta) \log (r / s)$ term is to keep $X_{n}$ bounded below, it is already bounded above by $\log s$

Claim $E\left(X_{n} \mid \iota_{1}, \quad, t_{n-1}\right) \leq \log r$ for all $n \geq 1$ The expectation means with respect to the probability measure $P_{\mathrm{r}}$ on $\Omega$

Proof Assume $d\left(w_{l_{n-1}} \quad w_{i_{1}} x, w_{i_{n-1}} \quad w_{i_{1}} y\right) \neq 0$ Then $E\left(X_{n} \mid l_{1}, \quad, l_{n-1}\right)$

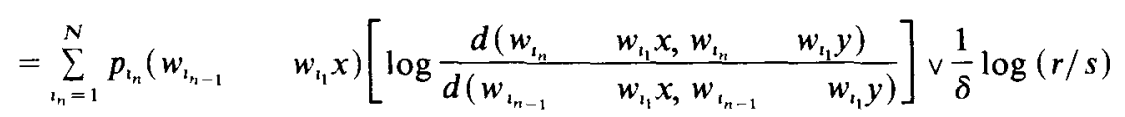

Assume the expression in brackets is $\geq(1 / \delta) \log (r / s)$ for each $t_{n}$ Then the hypothesis of the Theorem (take logarithms) implies that the above is $\leq \log r$ If for some $l_{n}$ the expression in brackets is $\leq(1 / \delta) \log (r / s)$ (which is negative), the fact that $p_{i_{n}} \geq \delta$ is easily seen to imply that the above is still $\leq \log r$ The claim is proved

Now let $D_{n}=X_{n}-E\left(X_{n} \mid l_{1}, \quad, l_{n-1}\right)$, so $D_{n}$ is a martıngale difference sequence, and $\left|D_{n}\right| \leq 2\left|X_{n}\right| \leq B$, say

Let $Y_{n}=\sum_{k=1}^{n}(1 / k) D_{k}$, so $Y_{n}$ is a martingale Now $E\left(Y_{n}^{2}\right) \leq B^{2} \sum_{k=1}^{\infty} 1 / k^{2}$ since $D_{k} \perp D_{l}$ for $k \neq l$ (because they are martingale differences) Thus $Y_{n}$ is an $L^{2}$-bounded martıngale, and so $Y_{n} \rightarrow$ a s Then by Kronecker's lemma,

$$
\lim _{n \rightarrow \infty} \frac{1}{n} \sum_{k=1}^{n} D_{k}=0 \quad \text { a s }
$$

Thus,

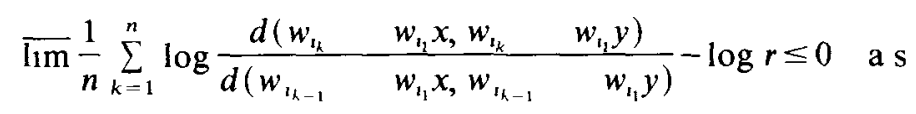

This telescopes to

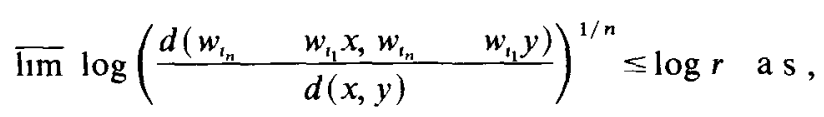


that is,

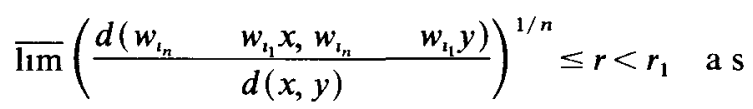

It is now easy to get from this that (1) of the conclusion holds, (11) follows immediately from (1)

LEMMA 3 Assume the hypotheses of the theorem Then for all $x, y$ in $X, P_{1}$ is absolutely continuous with respect to $P_{\mathrm{r}}$

Proof Let $P_{x}(E)=0$, and let $\varepsilon>0$ We shall show $P_{y}(E)<\varepsilon$ Let $r_{1}$ be as in Lemma 2

Let $\phi_{\imath}$ be the modulus of continuity of $p_{i}$, and let $\phi=\phi_{1} \vee \phi_{2} \vee \quad \phi_{N}$ Note $\phi$ is increasing

Claim $\sum_{k=1}^{\infty} \phi\left(r_{1}^{k} d(x, y)\right)<\infty$

Proof

$$
\begin{aligned}
\infty & >\int_{0}^{d(x, y)} \frac{\phi(t)}{t} d t=\sum_{k=1}^{\infty} \int_{r_{1}^{k} d(x, y)}^{r_{1}^{k-1} d(x, y)} \frac{\phi(t)}{t} d t \\
\geq & \sum_{k=1}^{\infty}\left(r_{1}^{k-1}-r_{1}^{k}\right) d(x, y) \frac{\phi\left(r_{1}^{k} d(x, y)\right)}{r_{1}^{k-1} d(x, y)} \\
& =\left(1-r_{1}\right) d(x, y) \sum_{k=1}^{\infty} \phi\left(r_{1}^{k} d(x, y)\right)
\end{aligned}
$$

which proves the claim

Now choose $m$ so large that $m>n_{\varepsilon / 2}$ from (1) of Lemma 2 and also $\sum_{k=m+1}^{\infty} \phi\left(r_{1}^{k} d(x, y)\right)<\delta / 2$

Let $\mathscr{A}_{\mathrm{n}}$ be the cylinder sets in $\Omega$ depending only on the first $n$ coordinates By a standard approximation result, there exist sets $A_{n} \in \mathscr{A}_{n}$ such that $E \subset \cup A_{n}$, the sets $A_{n}$ are disjoint and $P_{x}\left(\cup A_{n}\right)<(\varepsilon / 4)(\delta /(1-\delta))^{-m}$

Let $Q_{n}=\left\{\left(t_{1}, t_{2}, \quad\right) d\left(w_{t_{k}} \quad w_{t_{1}} x, w_{t_{k}} \quad w_{t_{1}} y\right) \leq r_{1}^{k} d(x, y)\right.$ for $\left.m \leq k \leq n\right\}, n \geq m$ Let $Q_{n}=\Omega$ for $n<m$ Thus $Q_{n} \in \mathscr{A}_{n}$ Let $Q=\bigcap_{n \geqslant 1} Q_{n}$ By Lemma 2(1), $P_{y}(\sim Q)<$ $\varepsilon / 2$ Let $n \geq m$ Now if $\left(l_{1}, l_{2}, \quad\right) \in Q_{n}$,

$$
\begin{aligned}
p_{t_{1}}(y) & p_{t_{n}}\left(w_{t_{n-1}} \quad w_{t_{1}} y\right) \\
\leq & p_{t_{1}}(x) \quad p_{t_{n}}\left(w_{t_{n-1}} \quad w_{t_{1}} x\right)\left(\frac{1-\delta}{\delta}\right)^{m} \\
& \times \prod_{k=m+1}^{n}\left[1+\frac{p_{t_{k}}\left(w_{t_{k-1}} \quad w_{t_{1}} y\right)-p_{t_{k}}\left(w_{t_{k-1}}\right.}{p_{t_{k}}\left(w_{t_{k-1}}\right.} w_{t_{1}} x\right) \\
\leq & p_{t_{1}}(x) \quad p_{t_{n}}\left(\begin{array}{ll}
w_{t_{n-1}} & \left.w_{t_{1}} x\right)\left(\frac{1-\delta}{\delta}\right)^{m}
\end{array} \prod_{k=m+1}^{n}\left[1+\frac{\phi\left(r_{1}^{k} d(x, y)\right)}{\delta}\right]\right.
\end{aligned}
$$

But

$$
\prod_{k=m+1}^{n}\left[1+\frac{\phi\left(r_{1}^{k} d(x, y)\right)}{\delta}\right] \leq 1+2 \sum_{k=m+1}^{\infty} \frac{\phi\left(r_{1}^{k} d(x, y)\right)}{\delta} \leq 2
$$


so

$$
p_{t_{1}}(y) \quad p_{t_{n}}\left(w_{t_{n-1}} \quad w_{t_{1}} y\right) \leq 2\left(\frac{1-\delta}{\delta}\right)^{m} p_{t_{1}}(x) \quad p_{t_{n}}\left(w_{t_{n-1}} \quad w_{t_{1}} x\right)
$$

When $n<m$, this holds trivially for any $\left(t_{1}, t_{2}, \quad\right)$

Thus,

$$
\begin{aligned}
& P_{y}\left(Q \cap A_{n}\right) \leq P_{u}\left(Q_{n} \cap A_{n}\right)
\end{aligned}
$$

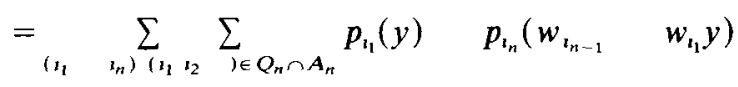

$$
\begin{aligned}
& \leq \sum_{\left(i_{1}, i_{n}\right)\left(t_{1} t_{2}\right.} \sum_{l \in Q_{n} \cap A_{n}} 2\left(\frac{1-\delta}{\delta}\right)^{m} p_{t_{1}}(x) \quad p_{t_{n}}\left(w_{t_{n-1}} \quad w_{t_{1}} x\right) \\
& \leq 2\left(\frac{1-\delta}{\delta}\right)^{m} P_{x}\left(A_{n}\right)
\end{aligned}
$$

So $P_{v}\left(\cup\left(Q \cap A_{n}\right)\right) \leq 2((1-\delta) / \delta)^{m} P_{x}\left(\bigcup A_{n}\right)$ since the $A_{n}$ 's are disjoint Now the right side is $<\varepsilon / 2$ by construction

Also $P_{v}\left(\bigcup\left(\sim Q \cap A_{n}\right)\right) \leq P_{v}(\sim Q)<\varepsilon / 2$, so we have then $P_{y}(E) \leq P_{v}\left(\bigcup A_{n}\right)<\varepsilon$

Proof of the Theorem Let $\left\{Z_{n}\right\}$ be the Markov process with transition probability $p$ as given in the introduction and such that $Z_{0}$ has distribution $\mu$ Then the process is stationary since $\mu$ is a stationary initial distribution, and is ergodic by Lemma 1 since $\mu$ is unique Let $f \in C_{c}(X)$, the continuous functions with compact support Then $\left\{f\left(Z_{n}\right), n=0,1, \quad\right\}$ 1s also stationary and ergodic [B, $\left.\mathrm{p} 119\right]$ Let

$$
B=\left\{\left(x_{0}, x_{1}, \quad\right) \in X^{\infty} \frac{1}{n+1} \sum_{k=0}^{n} f\left(x_{k}\right) \rightarrow \int f d \mu\right\}
$$

By the classical pointwise ergodic theorem, $P\left(\left(Z_{0}, Z_{1}, \quad\right) \in B\right)=1$

But

$$
\begin{aligned}
P\left(\left(Z_{0}, Z_{1}, \quad\right) \in B\right) & =\int P\left(\left(Z_{0}, Z_{1}, \quad\right) \in B \mid Z_{0}=x\right) d \mu(x) \\
& =\int P_{x}\left(\left(\iota_{1}, t_{2}, \quad\right)\left(x, w_{t_{1}} x, w_{t_{2}} w_{t_{1}} x, \quad\right) \in B\right) d \mu(x)
\end{aligned}
$$

Thus, for some $x_{0} \in X$,

$$
P_{x_{0}}\left(\left(l_{1}, l_{2}, \quad\right)\left(x_{0}, w_{l_{1}} x_{0}, w_{l_{2}} w_{t_{1}} x_{0} \quad\right) \in B\right)=1
$$

Let $G=\left\{\left(t_{1}, t_{2}, \quad\right)\left(x_{0}, w_{t_{1}} x_{0}, w_{t_{2}} w_{1_{1}} x_{0}\right) \in B\right\}$ Thus $P_{x_{0}}(G)=1$ and for $\left(\iota_{1}, \iota_{2}, \quad\right) \in G$,

$$
\frac{1}{n+1} \sum_{k=0}^{n} f\left(w_{\iota_{k}} \quad w_{t_{1}} x_{0}\right) \rightarrow \int f d \mu
$$

By Lemma 3, $P_{y}(G)=1$ for every $y \in X$ By Lemma 2(11), for every $y \in X$, there exists $H_{y}$ with $P_{v}\left(H_{3}\right)=1$ and for $\left(t_{1}, t_{2}, \quad\right) \in H_{y}$,

$$
\frac{1}{n+1} \sum_{k=0}^{n} f\left(w_{t_{k}} \quad w_{i_{1}} y\right)-f\left(w_{t_{k}} \quad w_{t_{1}} x_{0}\right) \rightarrow 0
$$


(note $f$ is uniformly continuous) Thus for $\left(l_{1}, t_{2}, \quad\right) \in G \cap H_{3}$,

$$
\frac{1}{n+1} \sum_{k=0}^{n} f\left(w_{i_{k}} \quad w_{i_{1}} y\right) \rightarrow \int f d \mu
$$

and $P_{y}\left(G \cap H_{y}\right)=1$

In the above, $G$ and $H_{v}$ depended on $f$ But since $C_{c}(X)$ is separable (since $X$ is $\sigma$-compact), we obtain that for each $y \in X$, there exists $G_{y}$ with $P_{y}\left(G_{y}\right)=1$ such that

$$
\frac{1}{n+1} \sum_{k=0}^{n} f\left(w_{i_{k}} \quad w_{l_{1}} y\right) \rightarrow \int f d \mu
$$

for each $f$ in a countable dense subset of $C_{c}(X)$, and then a $3 \varepsilon$ argument gives this for each $f \in C_{c}(X)$ Finally, since $\mu$ is a probability measure, it is easy to see (by Urysohn's lemma) that this holds for all $f \in C(X)$

Proof of Corollary 1 As in the proof of the theorem, let

$$
B=\left\{\left(x_{0}, x_{1}, \quad\right) \in X^{\infty} \frac{1}{n+1} \sum_{k=0}^{n} f\left(x_{k}\right) \rightarrow \int f d \mu\right\}
$$

Then

$$
\begin{aligned}
& P\left(\left(Z_{0}, Z_{1}, \quad\right) \in B\right) \\
& \quad=\int P\left(\left(Z_{0}, Z_{1}, \quad\right) \in B \mid Z_{0}=x\right) d \nu(x) \\
& \quad=\int P_{x}\left(\left(t_{1}, t_{2}, \quad\right)\left(x, w_{t_{1}} x, w_{t_{2}} w_{t_{1}} x, \quad\right) \in B\right) d \nu(x)
\end{aligned}
$$

But the integrand is 1 for each $x$ by the theorem

Acknowledgments We would like to thank Michael Barnsley for suggesting the problem, Marc Berger for pointıng out the work of Furstenberg and Kıfer, and Marc Berger and Jamie Goode for helpful discussions

\section{REFERENCES}

[B] L Breıman Probabilty Addison-Wesley (Readıng, Massachusetts, 1968)

[BD] M F Barnsley \& S Demko Iterated function systems and the global construction of fractals Prod $R$ Soc Lond A 399 (1985), 243-275

[BDEG] M F Barnsley, S Demko, J Elton \& J Geronımo Markov processes arısıng from function 1teration with place-dependent probabilities Preprint, Dec 1985

[BE] M F Barnsley \& J Elton Statıonary attractive measures for a class of Markov chains arısıng from function iteration Preprint, Aug 1985

[D] J L Doob Stochastic Processes Wiley (New York, 1953)

[DF] L Dubıns \& D Freedman Invarıant probabilitıes for certain Markov processes Ann Math Stat 32 (1966), 837-848

[DS] P Diaconıs \& M Shashahanı Products of random matrices and computer ımage generation Stanford U preprint (1984)

[FK] H Furstenberg \& Y Kifer Random matrix products and measures on projective spaces Israel $J$ Math 46 (1983), 12-32

[H] J Hutchinson Fractals and self-sımilarity Indiana U Journal of Math 30 (1981), 713-747

[K] S Karlın Some random walks arısıng in learnıng models Pacific J of Math 3 (1953), 725-756

[S] W F Stout Almost Sure Convergence Academic Press (New York, 1974) 